\title{
ECHOS
}

\author{
II \\ IMAGES NATIONALES
}


蒙馬特尔高地附近的 :

人、如女群起来保了大灿。

贲被服了土兵佣举行起义。
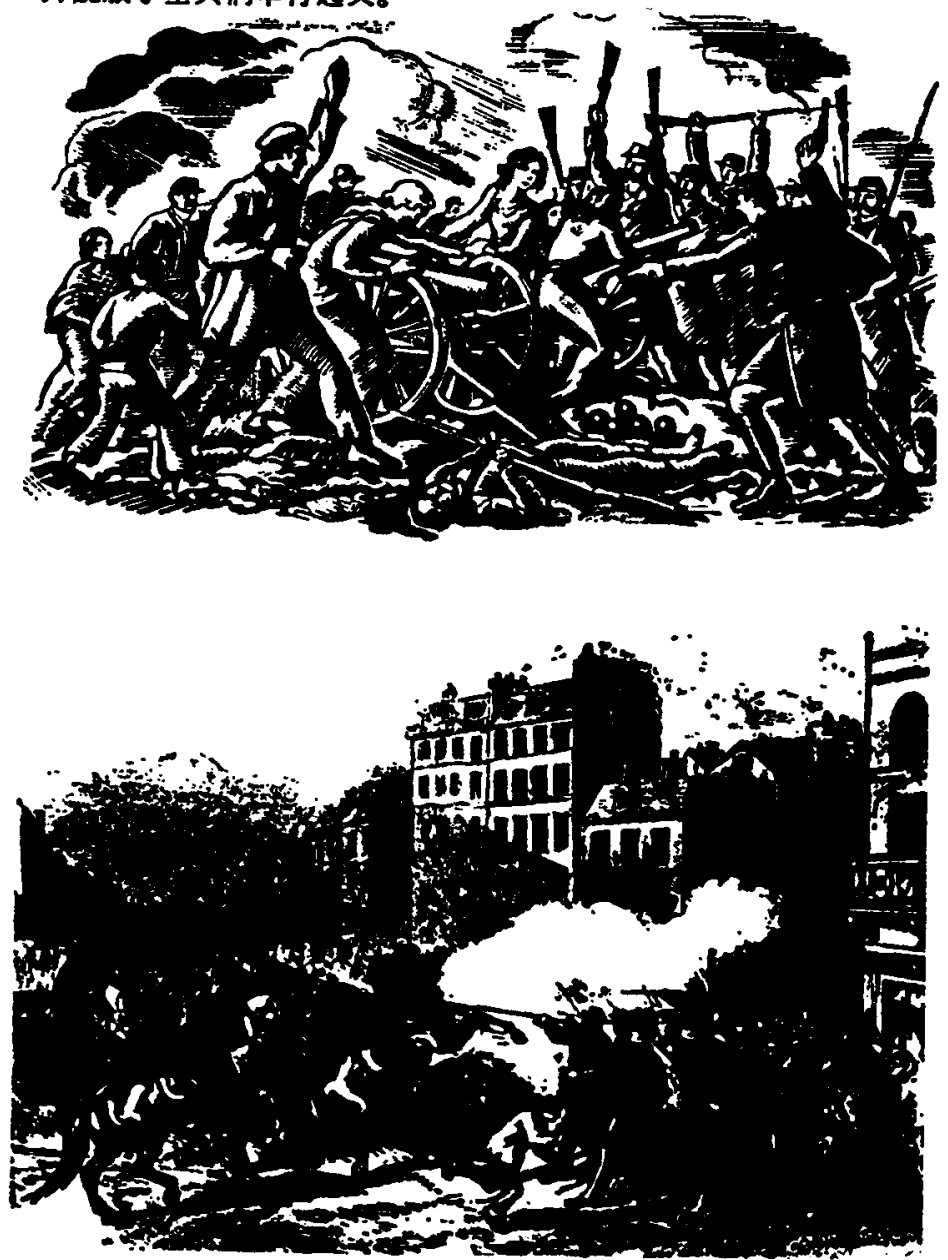

起又军闻反动軍队在 比答尔堨上战斗。 


\section{LA ROUMANIE}

"Je n'aime guère le mot influence qui ne désigne qu'une ignorance ou une hypothèse", écrivait Valéry. Or l'étude des dimensions et des répercussions internationales de la Commune de Paris s'est le plus souvent cantonnée à cet examen élémentaire des «influences». De la sorte, toute une problématique complexe, aux facettes multiples, vaste dans ses prolongements temporels, s'est trouvée réduite à un discours illustratif, celui qui est de rigueur lors des commémorations; elle se délaie dans des exposés linéaires, truffés de citations pêchées çà et là, dans la presse, dans les écrits de quelques contemporains, pimentés par la découverte d'un concitoyen parmi les participants à la Commune, augmentés de l'évocation automatique et si facile des agitations sociales qui accompagnèrent ou suivirent la Commune; on établit ainsi une concordance sans jamais faire la distinction entre les coïncidences et les répercussions. Et cependant quelques études sérieuses et rigoureuses ${ }^{1}$ démontrent l'intérêt et l'importance du sujet, et par là même la nécessité d'élaborer une véritable thématique.

Le choc de la Commune: voilà l'un des thèmes premiers, circonscrit dans le temps, mais étendu dans l'espace. Les connaissances, fragmentaires en ce domaine, sont surtout sectorielles et se limitent la plupart du temps au cas de quelques grandes puissances: Allemagne, Russie, Autriche-Hongrie... Mais l'élargissement géographique de l'enquête ne sert pas uniquement à accumuler les illustrations, il permet aussi de mesurer la complexité des répercussions internationales de l'événement. Ici l'exemple, réduit en apparence, de la Roumanie présente un double intérêt:

1) Le choc produit dans un état national récent, encore sous tutelle

1 Je pense notamment à l'ouvrage de Günter Grützner, Die Pariser Kommune. Macht und Karriere einer politischen Legende. Die Auswirkungen auf das politische Denken in Deutschland, Cologne et Opladen 1963; Klaus Meschkat, Die Pariser Kommune von 1871 im Spiegel der sowjetischen Geschichtsschreibung, Berlin 1965. 
des grandes puissances, en pleine effervescence nationale et sociale, à la recherche d'un modèle de développement et d'un régime politique.

2) Le choc produit au sein d'un peuple, dans une société profondément francophile, latine, où l'influence de chacun des "accidents» de l'histoire française a dès la fin du XVIIIe siècle profondément marqué les esprits avancés, dans un pays qui ne fut pas seulement en 1848 l'ultime frontière de la révolution européenne, mais qui subit directement le contrecoup du soulèvement parisien.

Fait significatif: on ne peut pas parler en Roumanie d'un impact immédiat des événements de 1871 . Les répercussions de la Commune se situent plutôt dans le sillage du choc premièrement provoqué par la guerre franco-prussienne, et découlent fondamentalement de l'effervescence catalysée par la chute de l'Empire et la proclamation de la République.

Dès son déclenchement, la guerre franco-prussienne a concentré sur elle l'attention de l'opinion publique roumaine. Elle a fait naître un puissant mouvement de sympathie pour la France qui a pris des proportions inhabituelles. Des comités d'aide à la France se constituent dans de nombreuses villes de Roumanie et parmi la population roumaine de Transylvanie. ${ }^{1}$ Ils se proposent de réunir des fonds pour l'armée française, par des collectes publiques, des souscriptions ouvertes dans la presse. Pour "sauver l'honneur de la nation française», des officiers roumains demandent à s'engager dans la légion étrangère; les volontaires affluent: s'il faut en croire la presse, plus de 900 Roumains se sont engagés dans l'armée française afin de combattre "pour la cause de la liberté»." Parmi eux, des étudiants qui poursuivent leurs études en France, de jeunes intellectuels nationalistes de Transylvanie, des militants radicaux ou des révolutionnaires romantiques qui avaient déjà été volontaires pour se battre aux côtés de Garibaldi, nombre de médecins, dont le général-major C. Davila, le chef du service de Santé de l'armée roumaine. ${ }^{3}$ Les lignes qu'adresse à celui-ci sa femme, apprenant que les troupes prussiennes sont arrivées devant Metz, sont révélatrices de l'état d'esprit d'alors: «les hordes barbares enva-

1 Cf. Stefan Manciulea, "Legăturile Blajului cu Franța în anii 1870-71", in: Transilvania, LXXVI (1945), no 1-2, pp. 50-58.

2 Baron Nicolae Vasilco, "Românii in Francia", in: Gazeta Transilvaniei, XXXIV (1871), no 32 du 24 mars, pp. 3-4.

3 Voir à ce sujet Sl. Leiderman, Răsunetul războiului franco-german din 1870 1871 in presa medicală română, Cluj 1938 (thèse pour le doctorat de médecine no 1283); G. Haupt et G. Barbu, "Participarea unor medici și studenți români.la războiul franco-german din 1870 și la Comuna din Paris", in: Studii si articole de istorie, t. II, Bucarest 1957, pp. 552-55; sur Davila, voir G. Z. Petresco, Charles Davila et l'influence française sur la médecine roumaine, Bucarest 1930. 
hissent la France du XIXe siècle et l'Europe ne bouge pas? Adieu Liberté!». Puis elle relate l'impression produite dans la capitale roumaine par les victoires allemandes: «les sympathies pour la France sont à ce point manifestes que le gouvernement s'en est inquiété jusqu'à juger nécessaire de consigner l'armée pendant trois jours consécutifs, les 7,8 et 9 août. L'indignation qui règne partout est immense. Tout le monde, jusqu'aux commerçants, parle à haute voix dans la rue, maudissant les Prussiens. Si vraiment la France emporte la victoire à Metz, même si ce n'est qu'une victoire partielle, celle-ci aura un très grand retentissement en Roumanie." ${ }^{1}$

Les succès de l'armée allemande ne font qu'attiser le mouvement francophile, qu'alimentent encore les attaques vigoureuses de la presse d'opposition contre la Prusse. Le gouvernement conservateur cherche à freiner cette agitation de plus en plus turbulente, qui tourne aux manifestations publiques; à la demande du Consul général prussien, il interdit de chanter la Marseillaise en public. ${ }^{2}$ Mesures qui ne provoquent pas d'incident diplomatique, car les représentants du gouvernement français se tiennent eux aussi sur la plus grande réserve, quand ils ne témoignent pas même d'une certaine hostilité envers ce mouvement francophile qu'ils jugent subversif. C'est que, constatant que les sentiments de sympathie manifestés par l'opinion publique roumaine "sont loin d'être partagés par le chef de l'Etat chez qui sans doute la communauté d'origine et les liens du sang sont plus forts que la reconnaissance qu'il nous doit et la conscience des véritables intérêts de la Roumanie», ceux-ci voient moins dans les mesures répressives l'expression de la germanophilie du prince régnant Carol de Hohenzollern et de son gouvernement conservateur, qu'une inquiétude légitime motivée par la détérioration de la situation politique intérieure. ${ }^{3}$ Le mouvement francophile revêt en effet les allures d'une manifestation d'hostilité à l'égard du jeune souverain, savamment alimentée et exploitée d'ailleurs par l'opposition libérale qui a été éliminée du pouvoir en 1869. Brandissant l'étendard antidynastique et germanophobe, cette opposition, stimulée par la guerre franco-prussienne, canalise à ses fins l'enthousiasme pro-français. ${ }^{4}$ D'où la réserve des diplomates français qui mettent l'agitation au compte des «rouges» (les libéraux), qu'ils considèrent comme des «agents professionnels à la solde

1 Elena General Petricari Davila, Din Viața și corespondenţa lui Carol Davila, Bucarest 1935, pp. 255-56.

2 Constantin Bacalbașa, București de altădată, 2e éd., Bucarest 1935, t. I, p. 24.

3 Ministère des Affaires étrangères, Archives diplomatiques, Agence et Consulat général de France à Bucarest, Correspondance politique, 1870, vol. 34.

4 B. Marinescu et S. Rădulescu-Zoner, "Le peuple roumain et la guerre francoprussienne de 1870-1871", in: Revue Roumaine d'Histoire, 1971, no 2, pp. 332-34. 
de la Russie", qu'ils accusent de profiter des sentiments authentiques d'hostilité du peuple roumain à l'égard de l'Allemagne pour semer l'anarchie et fomenter un complot contre le prince Carol. Craintes d'ailleurs partagées par le Quai d'Orsay. Dans les instructions au Consul général de France à Bucarest, le Ministre des Affaires étrangères précise la position du gouvernement impérial qui désapprouve toute action qui serait dirigée contre la dynastie, attire l'attention sur la nécessité de maintenir le calme et l'ordre interne en Roumanie et assure le souverain et son gouvernement de l'aide et de l'appui de la France.

En fait, le courant antidynastique est des plus hétéroclites; il comprend des groupes très divers, et des tendances divergentes: conservateurs séparatistes de Moldavie; partisans de l'ancien souverain A. I. Cuza, destitué en février 1866; ceux mêmes qui l'avaient destitué, les libéraux dirigés par I. C. Brătianu et qui avaient été les premiers partisans du souverain Hohenzollern; radicaux qui se situent à l'aile gauche du parti libéral, groupés autour de C. A. Rosetti, directeur du quotidien Românul; petits groupes enfin de républicains indépendants peu nombreux mais qui disposent d'une arme puissante, la presse satirique. Dans l'esprit des libéraux et des radicaux qui mènent en fait la campagne de presse contre le prince Carol, la victoire de Napoléon III ne peut qu'entraîner la chute du souverain. Exaltés par cette perspective et la tension qui règne dans le pays, ils sont prêts à passer au coup d'état. La révolte devait éclater simultanément dans six villes la nuit du 8 (20) août 1870 et profiter du soutien d'une partie de l'armée. Ajournée au dernier moment, la conspiration échoue à cause d'une action prématurée et isolée de la ville de Ploești. ${ }^{1}$ Le gouvernement riposte par une répression vigoureuse, qui n'atteint pourtant pas son but. L'échec n'a fait que stimuler le mouvement antidynastique qui, subissant le contrecoup des événements extérieurs, précise son orientation. L'écroulement de l'Empire en France est salué dans l'opposition avec vigueur: on attribue la responsabilité du désastre, de l'humiliation nationale à ce "régime odieux qui a dominé en France pendant 18 ans». ${ }^{2}$

Pour les vieux radicaux de 1848 , la France reste l'épicentre de tous les séismes révolutionnaires; ils voient dans l'avènement de la République l'éruption susceptible de précipiter une révolution européenne. Pour C. A. Rosetti, qui séjourne alors en France, le moment semble opportun pour la déclencher. En octobre 1870, il écrit à son ami Paul Bataillard que la France ne peut plus rien espérer des rois, qu'elle doit

1 Voir à ce sujet l'étude bien documentée de Gh. Cristea, "Conspiraţia <republicană> din august 1870", in: Studii, XXII (1969), n² 2, pp. 231-47.

2 Din viața si corespondenta lui C. Davila, pp. 258-59. 
faire appel aux peuples d'Europe pour les inciter à la révolte. L'entreprise lui semble aisée, et il s'engage pour sa part à soulever «l'Orient». ${ }^{1}$ Projet qui n'est en fait que l'expression exagérée de l'état d'esprit qui règne en Roumanie où la chute de Napoléon pose sous une forme aiguë le problème de la république et marque un moment important sinon un tournant dans l'évolution de l'agitation antimonarchique. Celle-ci s'intensifie, en même temps qu'elle revêt un caractère de plus en plus nettement républicain. Dès le début de 1871 , le ton est donné par la presse satirique républicaine, dirigée par un publiciste de talent, N. Orășanu, qui devient "l'homme du jour» et se taille une incontestable popularité par ses vers caustiques sur l'origine germanique du prince; on en fait des couplets à la mode. ${ }^{2}$ L'agitation républicaine ne se limite pas à la critique du souverain qui est sommé d'abdiquer; on appelle le peuple à la révolte, à recourir à la force en cas de nécessité. ${ }^{3}$ Ces pamphlets antimonarchiques trouvent un terrain favorable dans une opinion roumaine profondément émue et sensibilisée par la nouvelle de la défaite française.

Cet état d'esprit s'exprime dans les manifestations qui ont lieu le soir du 10 (22) mars 1871 à Bucarest. Ce jour, anniversaire de la naissance de l'empereur Guillaume ${ }^{e r}$, les membres de la colonie allemande de Bucarest se sont rassemblés dans la salle Slătineanu pour fêter leur victoire. Indignés, un très grand nombre d'habitants de Bucarest organisent une manifestation devant les locaux, conspuent les participants, les lapident, blessant même le consul général de Prusse, von Radowitz. La police reste impassible. On fait appel à l'armée pour disperser les manifestants dont le nombre grossit sans cesse et qui scandent les slogans: "Vive la république, vive la France, au Palais.» $\mathrm{Ce}$ n'est qu'à minuit que von Radowitz peut quitter la salle dévastée pour se rendre d'ailleurs au Palais demander réparation au nom du chancelier

1 Vasile Netea, C. A. Rosetti, Bucarest 1970, p. 301.

2 Le pamphlet de Orășanu intitulé Dicționarul politic sau epoca pruso-ciocoiască (Dictionnaire politique ou l'époque boyaro-prussienne) publié au début de 1871 est caractéristique de l'atmosphère de l'époque. C'est une violente satire antimonarchique où le roi par exemple est comparé au bourdon dans la ruche et où on lui prète le caractère et les mœurs d'un loup; mais le régime monarchique n'est pas éternel car, selon les vers d'Orășanu: "Où que l'on se tourne, on le voit souvent renversé par le peuple pour qui il était l'expiation de ses péchés." Le même Orășanu écrivait le 8 (20) mars dans le journal satirique Asmodeu: "L'un rit, l'autre pleure... L'un est le souverain, l'autre, le pauvre peuple. Veux-tu rire aussi pauvre peuple? Alors prends au souverain sa souveraineté."

3 Nous n'examinons pas ici tous les facteurs et tous les événements tels que par exemple l'affaire de la faillite Strousberg, scandale d'une concession de construction de chemins de fer, qui ont créé et alimenté l'atmosphère antimonarchique qui régnait dans le pays. 
Bismarck. L'atmosphère est si tendue que le prince Carol prend la nuit même la décision d'abdiquer. Seule l'intervention énergique du leader conservateur Lascăr Catargiu, appuyé par les représentants des "puissances garantes» qu'effraient les conséquences que pourrait avoir cette abdication, sauve la situation et contraint le prince à changer d'avis. ${ }^{1}$ Le cabinet libéral, présidé par Ion Ghica, au pouvoir depuis décembre 1870 , est accusé de complicité avec le mouvement républicain et démis de ses fonctions. Dès le lendemain 12 (24) mars, Lascăr Catargiu forme un gouvernement autoritaire qui s'appuie sur l'armée et dont le seul programme est de maintenir le prince. Les jours suivants, il déclare qu'il ne permettra pas que "la rue fasse la loi», dissout le Parlement qui lui est hostile le 15 (27) mars et fait traduire en justice le préfet de police libéral, Simion Mihălescu.

C'est dans une telle conjoncture que parvient la nouvelle de l'insurrection parisienne du 18 mars. Après le choc provoqué par la défaite française, par la capitulation, elle ne surprend personne ni n'est perçue comme révolution sociale. La signature précipitée de l'armistice par le gouvernement de défense nationale a soulevé une déception et une indignation générales. Dès le début de février 1871, les nouvelles en provenance de Paris signalaient la radicalisation rapide de l'opinion parisienne: "Tous demandent en France une nouvelle organisation, tous détestent et dédaignent l'égoïsme et l'emphase propres à une minorité de Français», écrit le 17 février 1871 un médecin roumain séjournant à Paris, le docteur Stefanesco. ${ }^{2}$ La Commune de Paris apparaît aux libéraux et aux radicaux roumains comme un mouvement républicain communal, et surtout comme un puissant sursaut populaire de résistance à l'envahisseur, seul capable de bouter hors les Prussiens.

$\mathrm{Au}$ début les nouvelles sont confuses, inexactes, contradictoires, car elles proviennent de sources indirectes, comme les dépêches d'agence ou les articles de la grande presse anglaise ou allemande. La presse roumaine n'a pas de correspondant permanent à Paris et les journaux de la Commune, notamment le Cri du Peuple et le Père Duchêne, ne parviennent à Bucarest qu'avec un retard considérable. Les feuilles roumaines abondent cependant en nouvelles en provenance de Paris,

1 Sur cet événement voir Monitorul Oficial al României, no 55, 1871, 11 (23) mars, p. 309; Aus dem Leben König Karls von Rumänien, Stuttgart 1894, t. II, pp. 174-77; Fr. Damé, Histoire de la Roumanie contemporaine, Paris 1900, pp. 226-29; Titu Maiorescu, Istoria contemporană a României, Bucarest 1925, pp. 30-31, et surtout l'étude de Gh. Cristea, "La guerre franco-allemande et le mouvement républicain de mars 1871 à Bucarest", in: Revue Roumaine d'Histoire, $1964, n^{\circ} 2$, pp. $277-91$.

2 Din viața și corespondența lui C. Davila, p. 279. 
que l'opinion publique attend avec impatience." "A Paris se décide le sort du monde libéral et par conséquent les Roumains doivent être intéressés par ces événements», proclame la presse libérale. La révolution communale qui s'accomplit le 18 mars est saluée comme celle de Paris «libre et libéré», même si son orientation politique et sociale demeure pour les libéraux roumains peu compréhensible, peut-être suspecte. Une vive polémique de presse s'engage début avril à propos de l'insurrection parisienne, lorsqu'on commence à entrevoir la tonalité socialiste que se donne la Commune. Les journaux conservateurs se déclarent ouvertement en faveur de Thiers et déclenchent une campagne virulente contre les insurgés. Quant à l'opposition, elle est divisée dans ses appréciations. Les libéraux, qui considèrent qu'une "lutte à mort a commencé à Paris entre la réaction vendue aux ennemis du pays et la démocratie dévouée à la patrie et à la nation», ${ }^{2}$ ne tardent pas à prendre position contre Versailles. Le nouveau quotidien radical à grande diffusion, Telegraful, qui paraît à Bucarest à partir du $1^{\mathrm{er}}(12)$ avril 1871 repousse, malgré toutes les réserves que lui inspirent les tendances socialistes de la Commune, les attaques des conservateurs et considère que "personne ne peut douter que Thiers est l'ennemi de la république», que le "sang répandu sous le prétexte du rétablissement de l'ordre est un sacrifice apporté à la monarchie». ${ }^{3}$ La condamnation non sans équivoque de Versailles n'implique pas pour autant un soutien aux Communards. La rédaction de Telegraful précise sa position: "Nous ne voulons pas nous associer à ceux qui, sans aucune réserve, condamnent la Commune proclamée à Paris, déclarant qu'elle est un instrument des anciens usurpateurs et tyrans de la France; tout au contraire, nous estimons que l'intention des insurgés était noble, qu'elle était patriotique et sublime, mais nous craignons que les fruits de la lassitude et des sacrifices qu'ils croient avoir apportés à l'autel de la patrie ne tombent en fin de compte entre les mains des ennemis mortels de la France. $\rangle^{4}$

Seule la presse satirique dirigée par N. Orășanu manifeste au mouvement parisien une sympathie sans réserve. Une lithographie publiée le 24 mars dans Ghimpele (La Griffe) intitulée «Derniers actes de la Comédie antique» est tout à fait caractéristique de cette tendance. Elle est de la plume de l'émigré révolutionnaire polonais H. Dembitzki,

1 Pour une analyse plus détaillée de l'écho provoqué par la Commune de Paris dans la presse roumaine, cf. P. Constantinescu-Jași, «Despre Comuna din Paris şi ecoul ei în presa contemporană din România», in: Studii, IV (1951), no 1, pp. 27-51

2 Telegraful, I (1871), n 14 du 16 avril, p. 1.

3 Ibid.

4 Ibid., 4 avril, p. 1. 
collaborateur de toutes les publications radicales roumaines et bulgares de l'époque: ${ }^{1}$ elle représente la Commune sous les traits d'un ouvrier qui brandit un marteau au-dessus de la tête des tyrans, des empereurs et du pape. Ceux-ci sont figurés sous la forme de reptiles et représentent, dit la légende, "une société pour l'exploitation des peuples par la grâce de Dieu" tandis que le prolétaire, symbole de la Commune, est désigné par l'inscription: "L'Internationale».

Les documents dont nous disposons ne permettent pas de percevoir la réaction de tous les secteurs de l'opinion publique. ${ }^{2}$ Une conclusion majeure se dégage pourtant des informations contradictoires parues dans la presse de l'époque et des commentaires qui les accompagnent, abondants en allusions aux réalités politiques roumaines. Pour l'opposition libérale et républicaine roumaine, la Commune apparaît dans un premier temps comme une nouvelle phase dans la lutte contre l'Empire et ses trahisons. Quant au contenu social, aux mesures sociales de la Commune, c'est l'incompréhension totale, même chez les radicaux qui y voient la réalisation d'idéaux généraux un peu abstraits et confus. Les idées socialistes sont encore largement étrangères à l'opinion publique roumaine qui en est restée, en matière sociale, aux doctrines romantiques utopiques de la révolution de 1848. En revanche, les conservateurs alertés dès le début, dénoncent immédiatement le "danger communiste" et entreprennent une campagne fort bien orchestrée ${ }^{3}$ qui atteindra assez vite son but, amortissant les effets du choc initial provoqué par la défaite française, dont on a vu pourtant l'ampleur.

1 Reproduit par T. Silanovska-Dimitrova dans son étude "Pe urmele lui Botev și Dembitzki in R.P.R.», in: Studii, II (1949), no 4, p. 101.

2 Cette courte étude ne se proposait pas de s'attarder sur tous les aspects de la question: ainsi entre autres problèmes celui de la participation des Roumains à la lutte de la Commune (qui a provoqué une plainte officielle du gouvernement français). Carol Ier notait dans ses mémoires le 25 mai (6 juin) 1871: "Le gouvernement français a communiqué au chargé d'affaires de la Roumanie que malheureusement, parmi les insurgés se trouvaient des Roumains qui avaient oublié leur dette de reconnaissance envers la France amicale et hospitalière. [...] ils se sont unis avec les communistes» (Memoriile regelui Carol I. al României, t. VI, p. 32). S'agit-il d'accusations gratuites du ministre Jules Simon? Le représentant diplomatique de Roumanie obtint la promesse de Jules Favre qu'il ferait transmettre au gouvernement roumain toutes les informations susceptibles de l'intéresser. Ces informations devaient probablement se trouver dans les dossiers 2 et 57 - 1871 du Ministère des Affaires étrangères de Bucarest intitulés "Participation des étudiants roumains de Paris aux troubles qui ont suivi la conclusion de la paix entre la France et l'Allemagne». Ces deux dossiers ont malheureusement disparu.

3 Voir l'article cité de P. Constantinescu-Jași, p. 42. 
Ce n'est que jusqu'à la mi-avril que l'insurrection parisienne sert de catalyseur encore à l'agitation républicaine qui connaît une dernière flambée dont l'ampleur frappe par exemple les cercles socialistes allemands. Ainsi lors d'un grand meeting de solidarité avec la Commune de Paris tenu à Hambourg le 13 avril, un orateur évoque l'importance des mouvements républicains croissants en Europe et cite, outre l'exemple de la France, ceux de l'Espagne et de la Roumanie. ${ }^{1}$ Le foyer d'agitation en Roumanie a retenu également l'attention de Marx, quoique sous un angle totalement différent: pour lui les menées du gouvernement tsariste contre l'Autriche sont susceptibles de ranimer la dangereuse question orientale s'il se crée en Roumanie une situation explosive: "Suivez bien attentivement, écrit-il à W. Liebknecht le 6 avril 1871, les événements dans les Principautés danubiennes. Si la révolution en France est temporairement vaincue - le mouvement ne peut y être réprimé que pour peu de temps - une nouvelle aventure guerrière s'ouvrira pour l'Europe à partir de l'Orient et la Roumanie servira de premier prétexte au tsar orthodoxe dans cette entreprise.» ${ }^{2}$

Ses craintes ont dû être bien vite apaisées: du jour au lendemain, la Roumanie cesse d'être un foyer d'agitation, l'élan républicain est brusquement brisé fin avril. Les élections municipales de Bucarest, dans lesquelles l'opposition libérale plaçait le plus grand espoir, se soldent pour elle par un retentissant échec. Les leaders de l'opposition doivent battre fortement en retraite et $\mathrm{C}$. A. Rosetti repart pour la France. Les conservateurs accréditent volontiers la thèse selon laquelle cette cuisante défaite et la disparition soudaine du mouvement républicain serait une conséquence fatale de l'adhésion qu'ils avaient apportée à la Commune de Paris. Carol Ier note le 19 avril (1 er mai) dans ses mémoires, à propos de l'échec des libéraux aux élections municipales de la capitale: "Les abominations de la Commune ont un peu calmé les

1 Der Sozialdemokrat, no 43 du 19 avril 1878, cité d'après B. S. Alexeev-Popov, Rabočij klass Germanii v dni Parižskoj Kommuny, Leningrad 1939, p. 156.

2 Georg Eckert (ed.), Wilhelm Liebknecht Briefwechsel mit Karl Marx und Friedrich Engels, La Haye 1963, p. 123. Certes la traduction des lettres de Marx n'est pas aisée; néanmoins, faut-il voir un contresens fortuit ou une erreur d'interprétation dans celle que proposent Ion Popescu-Puţuri et Augustin Deac dans leur brochure La Première Internationale et la Roumanie, Bucarest 1966, pp. 127-128? Nous citons le passage incriminé d'après ces deux auteurs: "Lorsque le 6 avril $1871 \mathrm{~K}$. Marx écrivait à Wilhelm Liebknecht: <il faut suivre avec attention les événements des Principautés danubiennes>, il se référait assurément aux manifestations révolutionnaires qui avaient eu lieu à Bucarest. Etablissant un rapport entre ces faits et la Commune de Paris, plein de confiance dans les forces révolutionnaires des pays roumains, Marx ajoutait: «Dans le cas où la révolution serait temporairement vaincue en France, là-bas (dans les Principautés danubiennes - A.D.) le mouvement ne pourrait être supprimé que pour un temps limités." 
esprits en Roumanie." ${ }^{\mathbf{I}}$ Le philosophe Titu Maiorescu, chef de file des jeunes conservateurs dissidents, donnera ultérieurement une explication semblable: "Le déclenchement de la Commune de Paris calma l'enthousiasme républicain en Roumanie.» ${ }^{2}$ Pour vérifier ces assertions, nous ne disposons que d'une source: la presse. A mesure que la Commune de Paris apparaît comme une révolution plus ouvrière, plus sociale, ou socialiste, la presse libérale, déroutée et inquiète, penche de plus en plus nettement vers la modération. L'esprit de classe l'emporte. La presse conservatrice, quant à elle, s'est servie de l'épouvantail communaliste pour intimider l'opinion publique et assimile les libéraux à leurs congénères parisiens, les accusant d'être des «agents des rouges de France». "Le mot Commune les a tous conquis, peut-être parce que de la Commune dérive le communisme", proclame l'organe officiel des conservateurs, le quotidien Pressa. ${ }^{3}$

Si la Commune déroute les libéraux, elle contribue largement à resserrer les rangs des conservateurs jusqu'alors divisés en groupes rivaux dont certains étaient parfois plus proches de l'opposition que du gouvernement. Sous le coup des événements de Paris, ils oublient pour un temps leurs discordes et se réunissent sous la bannière du gouvernement Catargiu pour apporter leur soutien sans réserve à la politique de répression. La "pétition-programme" élaborée fin avril 1871 par un groupe de conservateurs de Jassy (jusqu'alors en opposition avec le gouvernement) est à cet égard fort significatif. Signé aussi bien par le prince Grigore Sturdza, chef de la fraction des grands propriétaires terriens ultra-conservateurs que par les leaders des «junimistes» (jeunes conservateurs modernistes groupés autour de P. Carp et T. Maiorescu), ce document qui salue les mesures de répression prises contre les "rouges» affirme catégoriquement que sans une politique de force, d'intervention énergique, impitoyable «il y aurait eu dans notre pays un bouleversement dont les conséquences auraient été la copie à Bucarest de la Commune de Paris». ${ }^{4}$

Affirmations certes outrées. Mais elles illustrent parfaitement l'état d'esprit des forces de droite, des partisans sans réserve du gouvernement de Thiers qui devant la tournure «inquiétante» prise par les événements de Paris et l'offensive intérieure des radicaux sont passés dès avril 1871 à la contre-attaque, brandissant la menace rouge. Politique de force, action psychologique surtout destinée à conditionner l'opinion

1 Memoriile regelui Carol, t. VI, p. 29.

2 Titu Maiorescu, op. cit., p. 37.

a Sur cette campagne de presse qui visait à discréditer les libéraux, voir Gh. Cristea, "România și Comuna din Paris», in: Studii, XXIV (1971), no 2, pp. 213-16.

4 C. Bacalbașa, op. cit., t. I, pp. 26-27. 
publique par le moyen de la presse, en jouant la corde de la peur sociale. On l'attise par un tableau des horreurs de la Commune, présentée comme une entreprise de sauvagerie, de vols, de viols et de tuerie, et l'on justifie ainsi la répression sanglante des Versaillais. La presse libérale tente vainement de réfuter ces calomnies. C'est avec une certaine résignation que le journal Telegraful tente de dévoiler le cynisme et les méthodes de cette campagne si bien orchestrée: "Dans notre pays, la réaction se distingue avant tout en clamant le <De Profundis> parce qu'elle se sent suffisamment forte pour restaurer son passé honteux sur la tombe de tant de héros de la liberté... Il faudrait être dépourvu de bon sens pour ne pas voir que la réaction qui s'identifie pourtant à l'ordre, à la stabilité et au bonheur ne sème et ne récolte que désordre, instabilité, misère et incendies." ${ }^{1}$

Mais la psychose de peur est surtout créée grâce à un épouvantail: celui d'une vaste conspiration mondiale, fomentée par une association secrète qui compte des millions de membres, dont le siège est à Londres et dont le chef est un certain Dr. Marx. La Première Internationale, inconnue jusqu'alors en Roumanie, tient désormais la vedette dans la presse. ${ }^{2}$ On la présente comme une abominable subversion qui vient ruiner l'ordre établi, comme un complot international athée aux buts diaboliques. ${ }^{3}$ Les informations les plus fantaisistes circulent et le journal Telegraful, comparant l'attitude de la réaction à celle d'un noyé qui cherche son salut dans un fétu de paille, constate ironiquement: "La réaction a trouvé la branche à quoi s'accrocher, l'Internationale." ${ }^{4}$ Cette campagne qui devait originellement servir à créer une atmosphère d'hostilité envers l'opposition libérale déclenche un véritable mouvement d'hystérie collective qui n'épargne même pas le gouvernement qui fait appel à "tous les gens honorables, à tous ceux qui aspirent à la stabilité et au bien public" pour combattre et détruire les ramifications de cette funeste société, pour sauver l'humanité en péril. A l'automne 1871, le Premier ministre Lascăr Catargiu adresse une circulaire confidentielle aux préfets, ${ }^{5}$ où il écrit avec tout le sérieux que lui confèrent ses fonctions de chef du gouvernement et de ministre de l'Intérieur: «Si vous avez suivi les phases du désastre de la France et celles de l'incendie de Paris, vous avez pu apprendre qu'il existe une

1 Telegraful, I (1871), 26 mai, p. 1.

2 P. Constantinescu-Jași, art. cit., p. 50.

3 Il faut noter qu'en revanche la presse roumaine de Transylvanie tiendra un langage totalement différent et présentera des informations pleines de sympathie à l'égard de l'Internationale.

4 Telegraful, II (1872), no 37 du 12 mars, p. 1.

5 La photocopie de cette circulaire est reproduite dans Ion Popescu-Puturi et Augustin Deac, op. cit., p. 131. 
association de Communalistes qui a son siège à Londres et qui s'appelle l'Internationale. Autant les assertions de la presse que des informations confidentielles reçues de l'étranger m'ont confirmé dans la conviction qu'une section de cette association s'est également formée en Roumanie.» Après avoir rappelé les intentions atroces de ces comploteurs, il poursuit: «Comme je n'ai pu localiser la section roumaine, ni identifier ceux dont elle se compose», il leur demande de mettre tout en cuvre pour découvrir le siège de l'organisation et ses adhérents. L'enquête est demeurée sans résultat pour des raisons évidentes.

Si l'on voulait dresser un bilan provisoire du contrecoup de la Commune de Paris en Roumanie, on pourrait constater que celui-ci s'est soldé par un retour en force des conservateurs, par la création d'une psychose collective qui débouche sur une méfiance tenace à l'égard des idées socialistes qui va entraîner une longue résistance à leur pénétration, et sur le maintien de l'emprise des libéraux sur tous les courants d'opposition. Néanmoins des questions majeures restent posées. Pourquoi la situation explosive qui s'était créée en Roumanie en mars 1871 a-telle été aussi subitement désamorcée? Comment ce freinage brusque, ce revirement presque total opéré par les libéraux ont-ils pu se produire? Est-ce la conséquence d'un réflexe de classe déclenché par la crainte de la révolution prolétarienne parisienne qui a "tempéré le zèle et l'enthousiasme républicains, l'ardeur des libéraux à prendre le pouvoir", qui a éteint la braise du romantisme révolutionnaire de 1848 ? Cette explication passe-partout est peut-être trop commode. Ne faudrait-il donc pas élargir le champ d'investigation et tenir compte d'éléments déjà suggérés par Marx: le changement dans la conjoncture internationale où les rapports de forces ont basculé en faveur de la Russie tsariste? En d'autres termes, les libéraux roumains n'ont-ils pas pris conscience de la menace d'un danger extérieur lorsqu'ils ont renoncé à exploiter le moment favorable au renversement du prince Carol, après la dissolution du Parlement? N'ont-ils pas aussi refusé de compromettre la situation de leur pays privé du soutien de la France, menacé par les représailles de la Prusse, conjoncture qui risquait de le livrer à la Russie? 1

1 Gh. Cristea qui suggère cette explication (art. cit., p. 221, note 20) reproduit en note le rapport confidentiel envoyé de Berlin le 2 mai 1871 par P. P. Carp au sujet des conversations qu'il venait d'avoir avec Bismarck. Le chancelier aurait affirmé: «...Jusqu'à présent tout nous fait croire que le peuple [roumain] nous est hostile et, la haine provoquant la haine, vous comprendrez bien que la Prusse ne fera qu'user de justes représailles en se mettant du côté de vos ennemis. La Russie et la Turquie désirent la séparation et si l'occasion se présente, nous les aiderons à amener un changement, qui après tout, serait peut-être plus propre à favoriser votre développement..." 
L'exemple de la Roumanie où le choc de la Commune et de la situation internationale créée par elle ont joué le rôle d'un catalyseur négatif est-il un cas isolé? Ne peut-on déceler, au-delà de la situation apparemment sans issue d'un petit pays à la merci des fluctuations dans les rapports entre les grandes puissances, où les réflexes et les choix de politique intérieure sont conditionnés par la menace qui pèse sur une indépendance récente et fragile, des phénomènes plus profonds, plus généraux, qui se sont produits à l'échelle européenne sous des formes moins directement perceptibles, sous l'emprise de motivations différentes?

Sans apporter de réponse définitive, le cas ici étudié illustre la complexité du problème de l' «influence» de la Commune, remet en question la place privilégiée que lui accorde une historiographie classique comme ferment, comme levier de progrès révolutionnaire et socialiste, oblige à tenir compte des inhibitions, du traumatisme provoqué par un choc qu'ont su exploiter, à terme, les forces conservatrices. 